\title{
A comprehensive study of paediatric sepsis incidence
}

Lisa Mellhammar, Linnea Ollermark, Adam Linder, Department of Clinical Science,

University of Lund

\section{Conclusions}

We estimated the paediatric incidence of severe sepsis, even though based on small number of patients, the study design is

comprehensive.

When defining sepsis as an organ dysfunction and infection, which is more in accordance with the sepsis-3 for adults, the number were more than doubled suggesting the present definitions might gain of revision.

\section{Background}

Although sepsis is recognized as a major contributor to the burden of diseases in children, data on sepsis incidence are incomplete. Epidemiological studies are often based on either administrative data, which have shown poor agreement with clinical data, or are restricted to paediatric intensive care units, whereas sepsis might be treated in wards.

Sepsis definitions in adults have been revised due to increased insight in pathobiology, for example Systemic Inflammatory Response Syndrome, SIRS is no longer required.

Unfortunately, the pediatric sepsis

definitions remains unchanged since 2005.

\section{Objectives}

To estimate the incidence of severe sepsis in all paediatric clinics in a region. Furthermore, we calculated the incidences based on different sepsis definitions.

\section{Methods}

A retrospective review of medical records of children treated with intravenous antibiotics in all paediatric clinics in the region of Skåne, Sweden on 24 dates from July 2016 to July 2017 . The incidences were calculated based on the population $<18$ years at the year-end 2016 , which was 278773

\section{Results}

164 patients were included in the study of whom 142 were regarded with an infection, 7 with severe sepsis according to the paediatric sepsis definitions, and 15 with an infection and one organ dysfunction.

This is equivalent to a severe sepsis incidence of $38 / 100000$ person-years (95\% Cl 10-66/100 $000)$. When the SIRS was not required and when only one organ dysfunction was required, the incidence of severe sepsis rose to 82/ 100000 person-years (95\% Cl 40-123/100 000).
Infection

+1 Organ Dysfunction 82/ 100000

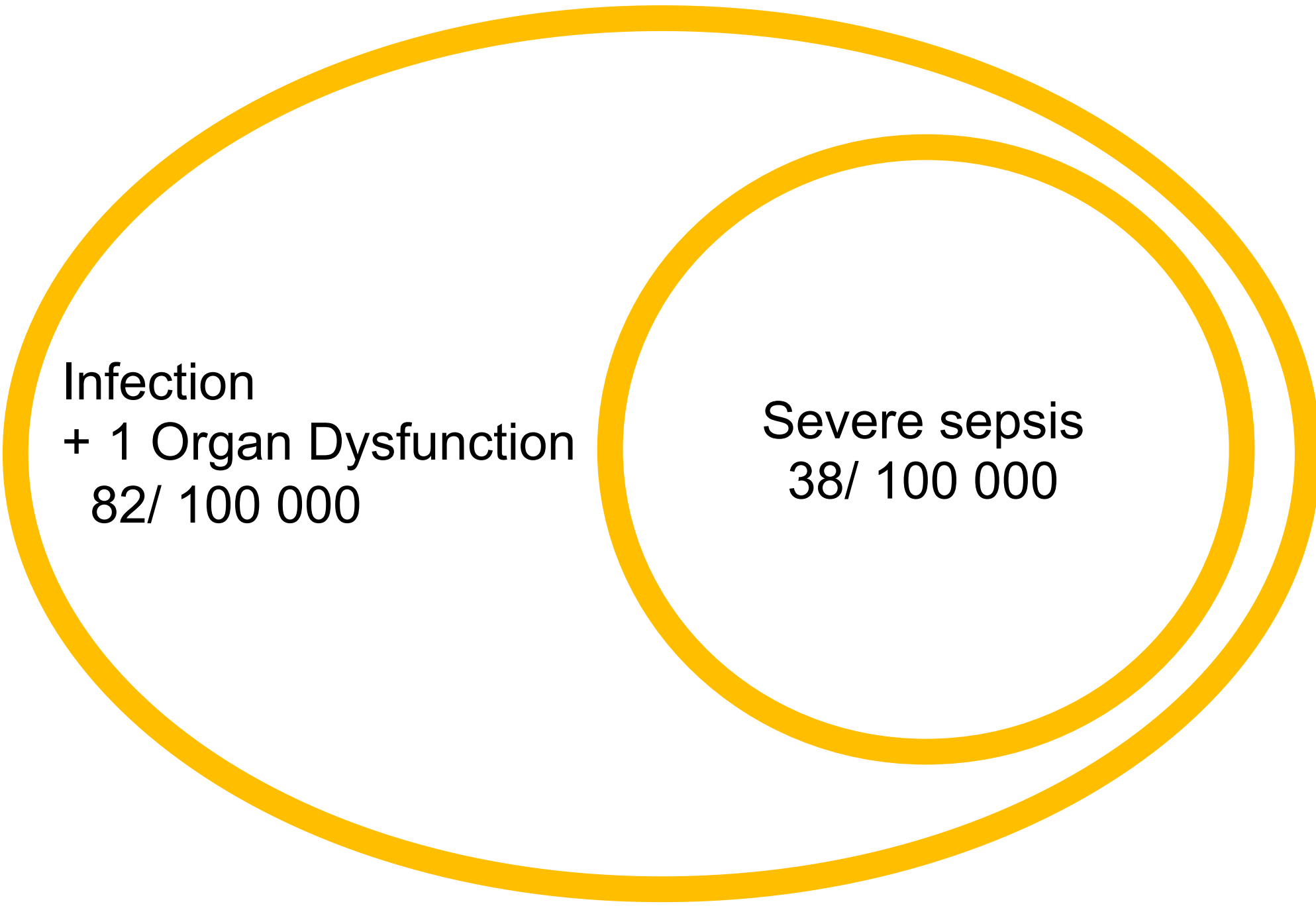

\section{References}

Fleischmann-Struzek et al. The global burden of paediatric and neonatal sepsis: a systematic review. Lancet Respir Med. 2018

Goldstein et al. International pediatric sepsis consensus conference: definitions for sepsis and organ dysfunction in pediatrics. Pediatr Crit Care Med. 2005

Singer et al. The Third International Consensus Definitions for Sepsis and Septic Shock (Sepsis-3). JAMA. 2016 